Conclusion Pesticide exposure is considerable among the farmers. The occupational health condi-tions presented by the farmers can be linked with their pesticide investigation. Although this study has presented some risk factors associated with general health symptoms, further investigation should look into specific pesticide-health correlation.

\section{A.5 EMERGENT ROLE OF EPIGENETIC BIOMARKERS OF PESTICIDES EXPOSURE: A CASE STUDY AMONG WOMEN OF CHILDBEARING AGE LIVING IN MEKNES (MOROCCO)}

\footnotetext{
${ }^{1,2}$ Aziza Menouni* ${ }^{*}$ Radu-Corneliu Duca, ${ }^{2}$ Manosij Gosh, ${ }^{1}$ Noura Zouine, ${ }^{1}$ llham Lhilali, ${ }^{1}$ Samir El Jaafari, 'Lode Godderis. 'Cluster of Competence 'Health and Environment', Moulay Ismail University, Meknes, Morocco; ${ }^{2}$ Health and Environment Unit, Faculty of Medicine, KU Leuven, Leuven, Belgium
}

\subsection{6/OEM-2019-EPI.10}

Pesticide exposure has been associated with acute and chronic adverse health effects. Current evidence supports that epigenetics may mediate these effects. DNA methylation (DNAm) is one of the broadly investigated epigenetic alteration. Therefore, to date, only limited human data is linking pesticide exposure to global DNAm alterations. Therefore, the aim of this study was to characterize pesticides exposure in women of reproductive age and investigate whether DNA methylation patterns were related to pesticides exposure level.

In a pilot study among 100 women from Meknes (Morocco), we measured 45 analytes (parent molecules and their metabolites) from three chemical families of pesticides: Organochlorines, organophosphates, pyrethroids, in urine and hair using HPLC/MS-MS and GC/MS. Commercial kits were used for quantification of 8-OHdG. We used LC/MS for measuring GSH. Pyrosequencing was used to measure Alu and LINE-1 methylation in DNA isolated from whole blood samples.

Pesticides are associated with genomic instability, which is proposed to be sensitive to nutritional intake and may also induce epigenetic changes. We evaluated the effect of life style and dietary intake on epigenetic instability in women. Our research prompts a re-thinking of the role of epigenetics on the understanding of the environmental exposure. We will then explore the role of epigenetic changes in the onset of cancer through the oxidative stress and DNA damage pathways.

\section{A.6 THE CARCINOGENICITY OF PESTICIDES USED IN NEW ZEALAND}

Andrea 't Mannetje*. Centre For Public Health Research, Massey University, Wellington, New Zealand

\subsection{6/OEM-2019-EPI.11}

Introduction Yearly over 3000 tonnes of pesticide active ingredients are applied in New Zealand agriculture. Since the 1980's, epidemiological studies have reported increased risks of lymphopoietic cancers in agricultural sectors with high pesticide use. Here we aim to estimate the number and total volume of currently used pesticides in New Zealand that are known or suspected human carcinogens, in order to inform interventions.

Methods For each of the pesticide active ingredients most commonly used in New Zealand, the carcinogenicity classification of three regulatory agencies (The New Zealand Environmental Protection Authority [NZ-EPA], the US Environmental Protection Agency [US-EPA], and the European Chemicals Agency [EU]) were extracted, as well as the classification of the International Agency for Research on Cancer (IARC) Monograph Programme. Total tonnes of active ingredients that are known or suspected human carcinogens was calculated for each classification.

Results None of the pesticides used in New Zealand are classified as known human carcinogens by any of the three regulatory agencies or IARC. Annually New Zealand uses 148-756 tonnes of active pesticide ingredients that are classified as suspected human carcinogens by the three regulatory agencies. If also including the pesticides classified by IARC as possible or probable human carcinogens, the upper estimate doubles to 1475 tonnes, representing half of the total volume of pesticide active ingredients used in New Zealand agriculture. The percentage and volume of active ingredients classified as suspected carcinogens by the three regulatory agencies was highest for the fungicides $(8 \%-60 \% ; 72-540$ tonnes), followed by herbicides (3\%-10\%; 60-200 tonnes), and insecticides (8\%, 16 tonnes).

Conclusions Although no known human carcinogens are used as pesticides, New Zealand's high use of pesticides that are suspected carcinogens requires a greater awareness of the presence of potential carcinogens in the agricultural sector and the development of an intervention strategy to reduce cancer risk.

\section{Reproductive Effects}

\section{B.1 MODELING INFERTILITY IN A COHORT OF CANADIAN TRADESWOMEN}

Nicola Cherry*, Jean-Michel Galarneau. University of Alberta, Edmonton, Canada

\subsection{6/OEM-2019-EPI.12}

Introduction With few female occupational cohorts, little is known about the contribution of work exposures to female infertility.

Methods A cohort of 888 tradeswomen across Canada was established (450 welders and 438 in the electrical trades) to examine effects on the fetus, with investigation of infertility a secondary objective. Women completed an extensive questionnaire at recruitment and follow-up questionnaires every sixmonths for up to 5 years. At each contact she was asked about attempts to get pregnant and barriers to conception. Fertility issues were identified both by self-report of failure to conceive for $\geq 12$ months and as the likelihood of conceiving in any month at risk. Determinants of infertility were examined in a Cox regression with time dependent covariates. Employment factors examined in each month were paid work, trade work and cumulative months in trade. Maternal age, prior conceptions, smoking, use of alcohol and BMI were examined as confounders.

Results 96 periods of infertility $\geq 12$ months were reported among 38 women from welding and 52 from electrical trades giving the risk of infertility for welders relative to 